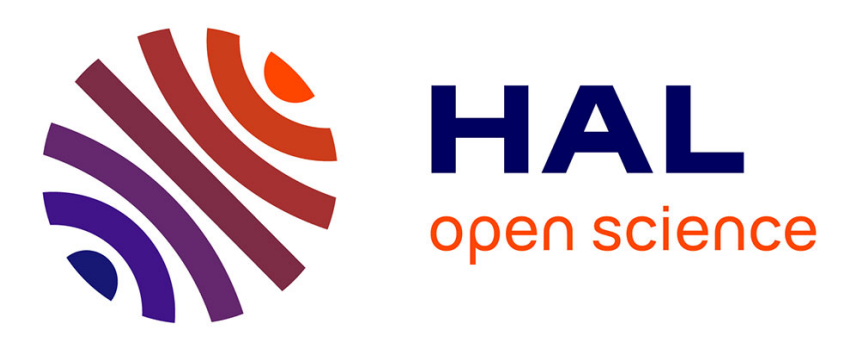

\title{
Transition from axisymmetric to three-dimensional turbulence
}

\author{
Zecong Qin, Aurore Naso, Wouter J.T. Bos
}

\section{To cite this version:}

Zecong Qin, Aurore Naso, Wouter J.T. Bos. Transition from axisymmetric to three-dimensional turbulence. Journal of Turbulence, 2021, pp.1-16. 10.1080/14685248.2021.1918700 . hal-03222604

\section{HAL Id: hal-03222604 \\ https://hal.science/hal-03222604}

Submitted on 10 May 2021

HAL is a multi-disciplinary open access archive for the deposit and dissemination of scientific research documents, whether they are published or not. The documents may come from teaching and research institutions in France or abroad, or from public or private research centers.
L'archive ouverte pluridisciplinaire HAL, est destinée au dépôt et à la diffusion de documents scientifiques de niveau recherche, publiés ou non, émanant des établissements d'enseignement et de recherche français ou étrangers, des laboratoires publics ou privés. 


\title{
Transition from axisymmetric to three-dimensional turbulence
}

\author{
Zecong Qin, Aurore Naso and Wouter J.T. Bos \\ Univ. Lyon, Ecole Centrale de Lyon, Univ. Claude Bernard, CNRS, INSA de Lyon, \\ Laboratoire de Mécanique des Fluides et d'Acoustique, F-69134, Ecully, France
}

\author{
ARTICLE HISTORY \\ Compiled May 10, 2021
}

\begin{abstract}
In purely axisymmetric turbulence sustained by a linear forcing mechanism, a stable, purely poloidal flow is observed when the toroidal component of the forcing is below a threshold. We investigate using numerical simulations whether this state persists when toroidal variations of the flow are continuously reintroduced and the forcing is purely poloidal. It is shown how the pressure-strain correlation allows the redistribution of the energy towards the toroidal component. A simple statistical model allows to capture the main physical effects on the level of the global energy balance. This model is then used to investigate the stability of the poloidal state for various toroidal-to-poloidal forcing strengths and different degrees of axisymmetry.
\end{abstract}

\section{KEYWORDS}

Axisymmetric turbulence; non-integer dimension; transition; statistical modeling

\section{Non-integer dimensions}

Most turbulent flows in nature are not statistically isotropic. In general body-forces associated with density differences, rotation, magnetic fields or imposed velocity gradients are present and induce anisotropy. Typical examples are strong rotation, or the presence of a strong magnetic field in conducting fluids, both leading to close to two-dimensional (2D) behaviours $[1,2]$. Even though asymptotically these flows tend to the perfect $2 \mathrm{D}$ limit $[3,4]$, in general the forces are not strong enough to perfectly two-dimensionalize them [5-7] so that the resulting dynamics are somewhere between $2 \mathrm{D}$ and $3 \mathrm{D}$ behaviours. Similar partial two-dimensionalization is observed in thin-layer turbulence [8-10], or in rotating Rayleigh-Bénard convection [11]. This partial twodimensionalization often complicates the analysis of realistic turbulent flows, since features characteristic of both 3D and 2D turbulence are entangled, and these flows are therefore not tractable by theoretical approaches applicable to either of the two asymptotic regimes.

A particular model-system which is also between two and three dimensions is purely axisymmetric turbulence. The axisymmetric Euler equations were first investigated in [12] to extend the successful statistical theories of two-dimensional turbulence [13-16] to a system in between two and three dimensions (and to the description of the flow within a vortex ring [17]). The theoretical predictions of this system were shown to be in qualitative agreement with experimental measurements of a stirred turbulent 
flow in a cylinder, which is only axisymmetric on average $[18,19]$. This success inspired further theoretical studies of the axisymmetric Euler system [20-23], and recently the first numerical simulations of strictly axisymmetric turbulence [24-26].

The most recent of these investigations $[26]$, in which a linear forcing protocol is used, showed that two very different types of flows can be observed in the axisymmetric system, depending on the forcing anisotropy. When only the poloidal components of the flow are forced, a purely poloidal flow is observed with features typical of twodimensional two-component (2D-2C) turbulence, such as an inverse energy cascade and coherent structures. It was shown that when a toroidal forcing is added, the purely poloidal flow remains stable and the toroidal flow absent, as long as the forcing anisotropy is sufficiently weak. Only when both forcing rates become of the same order, an abrupt transition is observed to a flow where both the poloidal and the toroidal components are non-zero. In this two-dimensional three-component (2D-3C) flow, a direct energy cascade is observed towards the small scales, and the coherent large scale structures are destroyed.

The assessment of the robustness of this critical transition is the main subject of the present work. More specifically, we want to answer the question whether the poloidal flow remains stable for a purely poloidal forcing, when pure axisymmetry is violated. For this, we will gradually re-introduce variations of the flow in the toroidal direction. In a sense, we will then investigate a continous transition between flows of different dimensionalities (axisymmetric [2D] to 3D). Such a procedure in which dimensionality is modified in a gradual way, was first introduced by Frisch et al., who investigated turbulence, and in particular the energy cascade, with a non-integer dimension [27, 28]. This change in dimension was applied directly to the statistical descriptors of turbulence, using two-point closure techniques. In direct numerical simulations, such a change of dimension does not seem straightforward, but recently a handle on this was found by applying spectral decimation on the Fourier-modes, thereby mimicking the mode and triad density of turbulence with a dimension between two and three $[29,30]$.

We do not proceed in the same way but rather consider flows varying slowly in the toroidal direction in such a way that the system can be continuously tuned from the axisymmetric limit to the three-dimensional limit: In practice, we will consider forced, partially axisymmetric turbulence with modified derivative operators. We will address two questions. First, how robust is the bifurcation from a purely poloidal (2D2C) flow to a three-dimensional three component flow when axisymmetry is not perfectly satisfied. Secondly, can we model this effect on the level of the energy balance, as we did for the purely axisymmetric case in [26]?

In the following section we introduce the model-equations. In Sec. 3 we describe the numerical methodology. Section 4 contains our numerical results. In Sec. 5 we attempt to model the system and show how the pressure term allows to redistribute energy between the components of the system. Section 6 concludes the manuscript.

\section{Notations and equations}

We consider a turbulent flow in cylindrical geometry. The height of the cylinder is $L$ and the radius $R$ (see Fig. 1). The axis is in the $z$-direction, the radial direction is $r$ and the azimuthal direction $\theta$. In [26] we considered the dynamics of purely axisymmetric turbulence in such a geometry. In particular, we considered the influence of the forcing anisotropy on the dynamics using a linear forcing protocol and varying the relative 


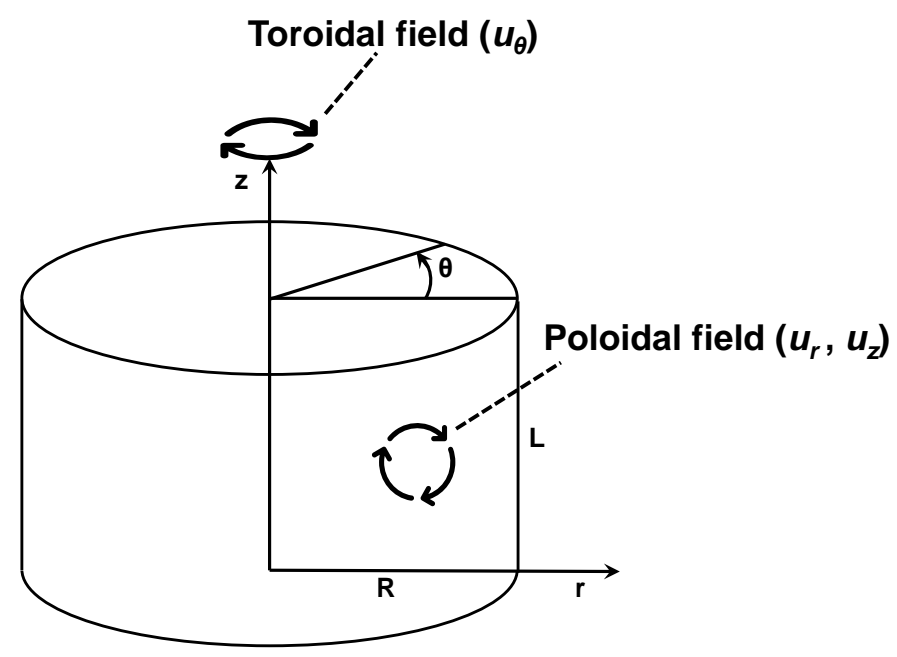

Figure 1. The cylindrical domain considered in the present investigation. The height of the domain is $L$, the radius $R$. The toroidal coordinate is indicated $\theta$ and the poloidal coordinates $r, z$.

poloidal and toroidal forcing-strengths. We assess in the present investigation whether the flow remains purely poloidal when the forcing is purely poloidal and a small amount of non-axisymmetry is introduced into the system. The way we do this will now be described. We consider the Navier-Stokes equations in cylindrical coordinates and apply them to a flow varying slowly in the azimuthal direction, $\mathbf{u}=\mathbf{u}(r, \alpha \theta, z, t)$ and $p=p(r, \alpha \theta, z, t)$, where $\mathbf{u}$ and $p$ are the velocity and pressure fields, respectively, and $\alpha$ is a parameter in the $[0,1]$ interval. This amounts to imposing a characteristic lengthscale in the toroidal direction $L_{\theta}=\alpha L_{r, z}$, where $L_{r, z}$ is the characteristic length in the poloidal (radial and axial) directions. The flow is strictly axisymmetric (2D) when $\alpha=0$, and three-dimensional when $\alpha=1$. Under these conditions and after a change of variables $\left(\theta \rightarrow \theta^{\prime}=\alpha \theta\right)$, the Navier-Stokes equations write:

$$
\begin{aligned}
& D^{(\alpha)}\left[u_{r}\right]=f_{r}+\nu \Delta_{r}^{(\alpha)} u_{r}-\partial_{r} p+\frac{u_{\theta}^{2}}{r} \\
& D^{(\alpha)}\left[u_{z}\right]=f_{z}+\nu \Delta_{z}^{(\alpha)} u_{z}-\partial_{z} p \\
& D^{(\alpha)}\left[u_{\theta}\right]=f_{\theta}+\nu \Delta_{\theta}^{(\alpha)} u_{\theta}-\frac{\alpha}{r} \partial_{\theta} p-\frac{u_{\theta} u_{r}}{r},
\end{aligned}
$$

where the components of an externally imposed volume force are indicated by $f_{i}, \nu$ is the kinematic viscosity, and the ' have been omitted for the sake of simplicity. The operator $D^{(\alpha)}[g]$ is defined as

$$
D^{(\alpha)}[g]=\partial_{t} g+u_{r} \partial_{r} g+u_{z} \partial_{z} g+\alpha \frac{u_{\theta}}{r} \partial_{\theta} g
$$

$\Delta_{i}^{(\alpha)}$ are the three components of the cylindrical vector Laplacian, where, as in $D^{(\alpha)}[g]$, all azimuthal derivatives are multiplied by $\alpha$ or $\alpha^{2}$ according to their order. Roughly speaking, intermediate values of the parameter $\alpha$ between 0 and 1 correspond to dimensionalities of the Navier-Stokes equations between 2 and 3 . 
Incompressibility is ensured by the relation

$$
r^{-1} \partial_{r}\left(r u_{r}\right)+\partial_{z} u_{z}+\alpha r^{-1} \partial_{\theta}\left(u_{\theta}\right)=0 .
$$

As already mentioned, the above system represents the Navier-Stokes equations for $\alpha=1$ and axisymmetric flow for $\alpha=0$. In between these two values, the flow is expected to show a transition between the two regimes. It is this transition that we will try to characterize in the following ${ }^{1}$.

We will thereafter focus on the energy balance, and distinguish between the toroidal and poloidal energy components, respectively denoted as

$$
E_{T}=\frac{1}{2}\left\langle u_{\theta}^{2}\right\rangle, \quad E_{P}=\frac{1}{2}\left\langle u_{r}^{2}+u_{z}^{2}\right\rangle .
$$

Their ratio is denoted by

$$
\gamma=\frac{E_{T}}{E_{P}} .
$$

The energy balance derived from the system (1-5) formally writes:

$$
\begin{aligned}
\frac{d E_{P}}{d t} & =P_{P}-\epsilon_{P}+\Pi+T, \\
\frac{d E_{T}}{d t} & =P_{T}-\epsilon_{T}-\Pi-T,
\end{aligned}
$$

where the injection of energy is given by

$$
P_{P}=\left\langle u_{r} f_{r}+u_{z} f_{z}\right\rangle, \quad P_{T}=\left\langle u_{\theta} f_{\theta}\right\rangle
$$

and the dissipation rates

$$
\begin{aligned}
\epsilon_{P} & =-\nu\left\langle u_{r} \Delta_{r}^{(\alpha)} u_{r}+u_{z} \Delta_{z}^{(\alpha)} u_{z}\right\rangle \\
\epsilon_{T} & =-\nu\left\langle u_{\theta} \Delta_{\theta}^{(\alpha)} u_{\theta}\right\rangle
\end{aligned}
$$

The other two terms are redistributive terms which allow the energy to be exchanged between the toroidal and poloidal energy components. The quantity

$$
T=\left\langle\frac{u_{\theta}^{2} u_{r}}{r}\right\rangle
$$

is a transfer term and the pressure term is given by

$$
\Pi=\alpha\left\langle\frac{u_{\theta}}{r} \frac{\partial p}{\partial \theta}\right\rangle .
$$

\footnotetext{
${ }^{1}$ Another interesting limit is $\alpha \rightarrow \infty$. Indeed in this limit the system might also tend to an axisymmetric system, since all modes with variations in the $\theta$ direction will attenuate through diffusion so rapidly that variations are smoothened out. We have not checked this conjecture and focus here on the range between $\alpha=0$ and $\alpha=1$ where we know the precise behavior of the limiting cases.
} 
It is in particular this last term on which we will focus. Indeed, in our previous investigation [26] it was absent from the energy balance, since $\alpha$ was set to zero. The magnitude of this term will therefore (at least partially) determine how the energy balance will be affected if the flow is not strictly axisymmetric.

\section{Numerical method}

\subsection{Numerical setup}

We carry out simulations in a cylindrical geometry with an aspect ratio of $L / R=1.7$, and a radius $R=\pi$. Two values of the kinematic viscosity are used, $\nu=0.01$ and $\nu=0.001$, respectively. In a recent investigation of linearly forced isotropic turbulence at low Reynolds numbers in a tri-periodic cubic domain [31], we showed that the control parameter determining the onset of turbulence in such a system is

$$
R e=\frac{c_{P} L^{2}}{\nu},
$$

where the lengthscale $L$ was chosen equal to $L_{D} / 2 \pi$, with $L_{D}$ the domain size and $c_{P}$ the forcing-strength. The present configuration is obviously different, but we still define the Reynolds number according to (15), with $L$ the cylinder height. This gives not necessarily the same value for the critical value of the Reynolds number as in the isotropic case, but this is not the subject of the present investigation. The values of $R e$ in our simulations, using the above parameters with the two values of viscosity, are $R e=1141 ; R e=11410$.

The numerical code integrates the Navier-Stokes equations in cylindrical geometry and is based on a pseudo-spectral Fourier-Chebychev decomposition. The radial $(r)$ and axial $(z)$ directions of the cylindrical computational domain are discretized using Chebychev polynomials, while the toroidal $(\theta)$ direction is decomposed by Fourier series ensuring the periodicity condition. A projection scheme is used to solve the coupling between pressure and velocity [32]. An originality of the code [33] lies in the treatment of the azimuthal direction: the whole diameter $-R \leq r \leq R$ (not only the radius) is discretized with an even number of radial Gauss-Lobatto collocation points. This approach [34] avoids problems commonly encountered on the axis, $r=0$ in numerical simulations performed in cylindrical coordinates. The price to pay is that for the azimuthal discretization no fast-Fourier solver exists, which makes this approach prohibitively expensive for very high azimuthal resolutions. The precise formulation of the full numerical method and its convergence properties can be found in [33]. The modifications allowing to take into account the transition from axisymmetric to three dimensional turbulence, as well as their validation, are detailed in [35]. For $\nu=$ 0.01, the resolution of the simulations in the radial, vertical and azimuthal directions, respectively, is $N_{r} \times N_{z} \times N_{\theta}=190 \times 181 \times 80$ gridpoints, and the time step is set to $\Delta t=5 \times 10^{-4}$. Simulations with different values of $\alpha$ varying from 0 (strictly axisymmetric) to 1 (normal three-dimensional case) are carried out, namely $\alpha=0$, $0.05,0.1,0.15,0.2,0.25,0.3,0.4,0.5,0.6,0.7,0.8,0.9,1$.

With these settings, the attainable large-scale Reynolds number based on the kinetic energy, defined as $\operatorname{Re}_{E} \equiv L \sqrt{2 E / 3} / \nu$, is about 300 for $\nu=0.01$, which is rather low. In particular the case $\alpha=0$ is challenging, since due to the inverse cascade that we have evidenced in this case [26], the energy at the large scales increases importantly. The numerical resolution required to simulate such a flow therefore needs 
to be substantially larger than for the three-dimensional or partly three-dimensional flows. In two-dimensional turbulence, without walls, the dissipation tends to zero for large Reynolds numbers. A steady state in linearly forced two-dimensional turbulence will therefore contain a kinetic energy which becomes asymptotically large if the viscosity tends to zero. In the present case, where solid boundaries are present, dissipation is present in this limit depending on the asymptotic scaling of the turbulent boundary layer thickness. This thickness is still a subject of debate [36,37]. We do not go further into this debate, but have observed that it is computationally very challenging to obtain converged statistics in a steady state in the poloidal $\alpha=0$ limit. Even at the relatively low Reynolds numbers considered, simulations could be carried out for a limited time only and the modified Laplacian $\Delta_{i}^{(\alpha)}$ in expression (1) was replaced by the normal Laplacian $\Delta_{i}^{(1)}$ in these direct numerical simulations (DNS). The non-integer dimension $\alpha$ therefore affects only the nonlinear and pressure terms in these DNS.

In order to investigate higher Reynolds number dynamics and to be able to use the modified Laplacian $\Delta_{i}^{(\alpha)}$, we also carried out simulations based on the spectral vanishing viscosity method (SVV) $[38,39]$. This method, of the Large Eddy Simulation type, allows to attain higher Reynolds numbers. The SVV operator can be expressed as:

$$
\nu \Delta_{S V V}^{(\alpha)} \equiv \nu \Delta^{(\alpha)}+\nabla^{(\alpha)} \cdot\left(\varepsilon Q \nabla^{(\alpha)}\right),
$$

where $\varepsilon$ is the additional viscosity and $Q$ is a scale-dependent coefficient which becomes larger at smaller scales. The SVV method consists in enhancing the dissipation for smaller scale, in order to allow for numerical convergence at high Reynolds numbers. Starting from the definition provided in Eq. (4), the SVV gradients and Laplacian are modified as in Eq. (1) to take into account the influence of $\alpha$ on the derivatives, i.e., first- (resp. second-) order derivatives are multiplied by $\alpha$ (resp. $\alpha^{2}$ ).

We verified that at $\nu=0.01$ the results of the SVV method and of the DNS concord. We will for instance show in Fig. 5 that the results for $\gamma$ are consistent between the two methods. We also present in the following results using this method for a viscosity $\nu=0.001$, therefore corresponding to a 10 times larger value of the Reynolds number, $R e=11410$, and using the modified Laplacian $\Delta_{i}^{(\alpha)}$. At this Re value it appeared impossible using the available numerical resources to reach a steady state for $\alpha=0$. Consequently, the SVV simulations are presented for $\alpha \geq 0.1$.

\subsection{Linear forcing}

The choice of the forcing protocol is important in the investigation of transitions. We use here a linear forcing protocol $[40,41]$, where the forcing strength is proportional to the energy in certain or all modes constituting the velocity field. In axisymmetric turbulence, we can introduce an anisotropic linear forcing

$$
\boldsymbol{f}=c_{r} u_{r} \boldsymbol{e}_{r}+c_{z} u_{z} \boldsymbol{e}_{z}+c_{T} u_{\theta} \boldsymbol{e}_{\theta} .
$$




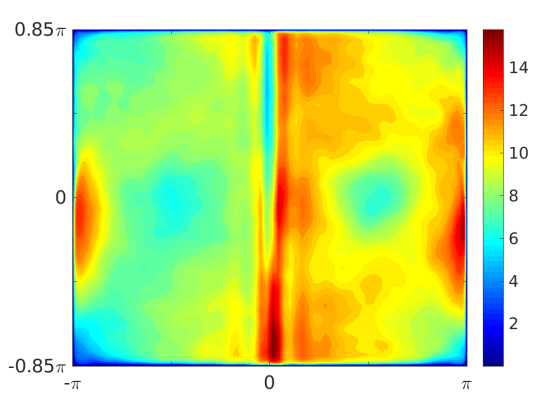

(a)

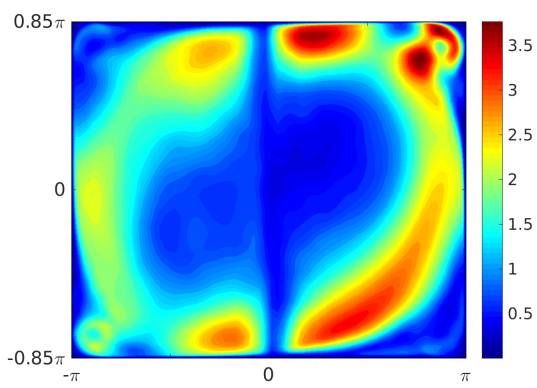

(c)

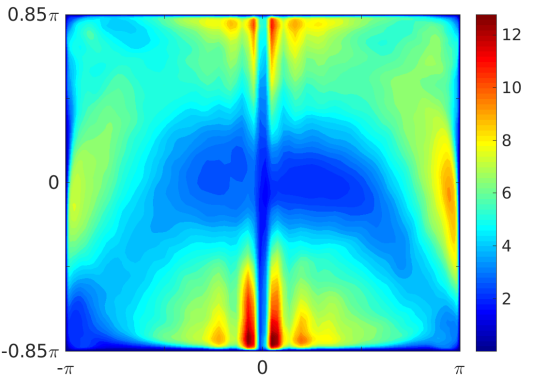

(b)

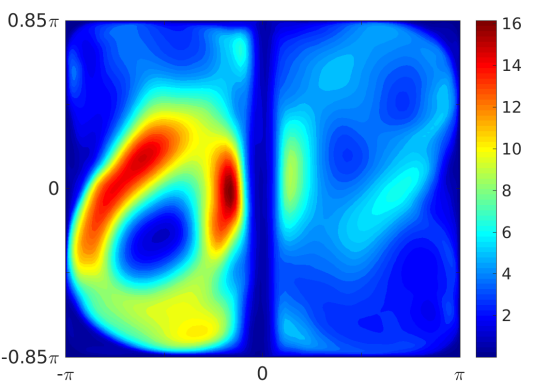

(d)

Figure 2. Typical instantaneous visualizations of the kinetic energy in poloidal planes for SVV flows at $R e=11410$ : (a) $\alpha=1$; (b) $\alpha=0.5$; (c) $\alpha=0.2$; (d) $\alpha=0.1$.

Solutions, $u_{r}=0, u_{z}=0$ or $u_{\theta}=0$ are all compatible with this forcing. The forcing is applied in the poloidal plane only, $c_{T}=0$ and $c_{r}=c_{z}=c_{P}$ so that

$$
\boldsymbol{f}=c_{P}\left(u_{r} \boldsymbol{e}_{r}+u_{z} \boldsymbol{e}_{z}\right)
$$

The forcing parameter is set to $c_{P}=0.4$.

Recalling the fact that in this limit the $\alpha=0$ axisymmetric flow does not generate any toroidal component [26], our choice of setting $c_{T}$ to zero will allow to see for which value of $\alpha$ this purely poloidal flow becomes unstable. This forcing is different from the spectral-band forcing used in [26], as it is applied to all scales of the velocity field, but both forcing schemes have a linear behaviour. The present forcing implementation is indeed more convenient with the here-used numerical method, in which wavenumbers cannot be easily defined in the poloidal plane due to the mixed Fourier-Chebychev decomposition.

\section{Numerical results}

\subsection{Flow visualizations}

Typical instantaneous visualizations of the kinetic energy in the poloidal plane are shown in Fig. 2 for four different values of $\alpha$ and a Reynolds number $R e=11410$. The visualizations show that different types of flow structures are observed. It is however not straightforward to characterize the flow or to discern particularities which are very much $\alpha$-dependent. 


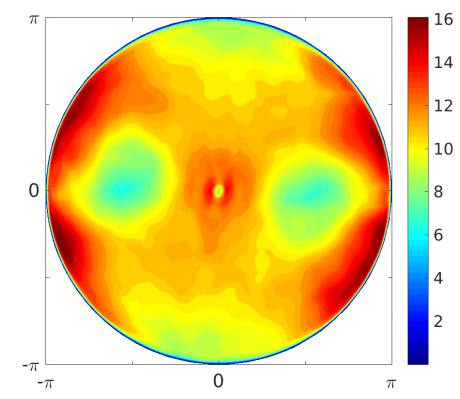

(a)

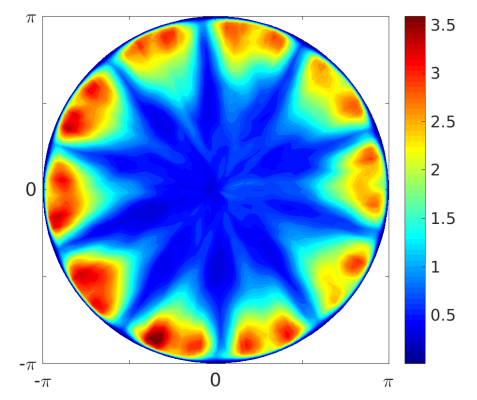

(c)

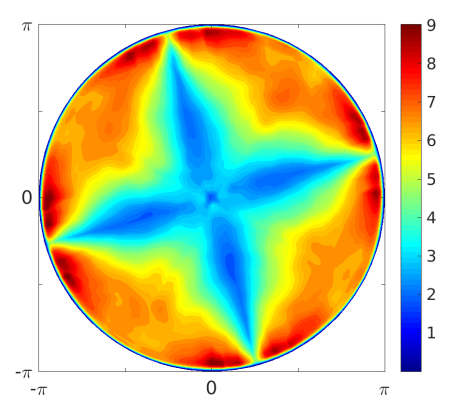

(b)

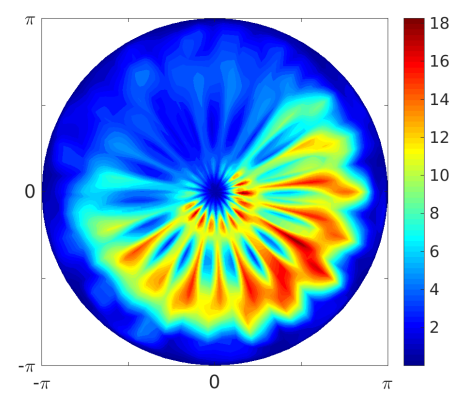

(d)

Figure 3. Typical instantaneous visualisations of the kinetic energy in a horizontal plane at mid-height of the cylinder, for the SVV simulations at $R e=11410$ : (a) $\alpha=1$; (b) $\alpha=0.5$; (c) $\alpha=0.2$; (d) $\alpha=0.1$.

This is quite different for the visualizations in Fig. 3 where we show kinetic energy patterns in the horizontal center-plane through the cylinder for the SVV simulations at $R e=11410$ and four values of $\alpha$ (the qualitative features of DNS and SVV at $R e=100$ are very similar). It seems that in the 3D limit $(\alpha=1)$ the flow exhibits a periodic behaviour with a two-cell structure in planes perpendicular to the cylinder axis. The presence of a small number of large scale structures is not surprising in this case, since our forcing drives all flow scales, including scales of the size of the domain. In the von Kármán flow, for instance, where the flow is driven at a scale of the same order as the domain size by rotating impellers, the mean-flow also exhibits a double cellular structure [42].

Varying $\alpha$ changes the frequency of this cellular structure with an approximate dependence proportional to $\alpha^{-1}$. Indeed, for $\alpha=0.5$, four structures are observed, $\alpha=0.2$ yields 10 cells and for $\alpha=0.1,20$ cells can be observed. This is a direct consequence of the definition of $\alpha$ (indeed, the characteristic length in the toroidal direction $l_{\theta} \sim \alpha^{-1}$ ).

\subsection{Time evolution of energy and dissipation}

In Fig. 4(a) we show the time signal of the kinetic energy averaged in the computational domain. A very distinct behaviour is observed for the different cases. In particular for $\alpha=1$, an almost periodic behavior is observed, leading to large variations around the steady state value. The other simulations show less important fluctuations during the steady state. The dissipation, shown in Fig. 4(b), follows this behavior, although the time signals naturally display higher frequencies. 


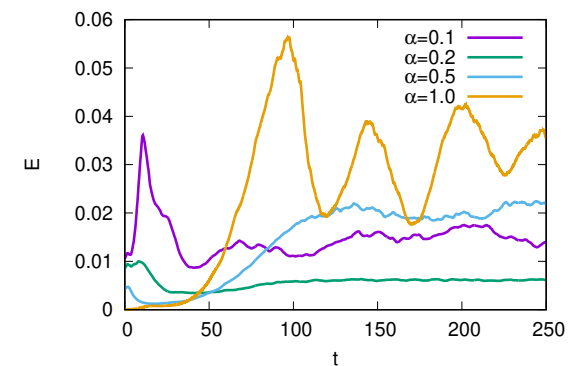

(a)

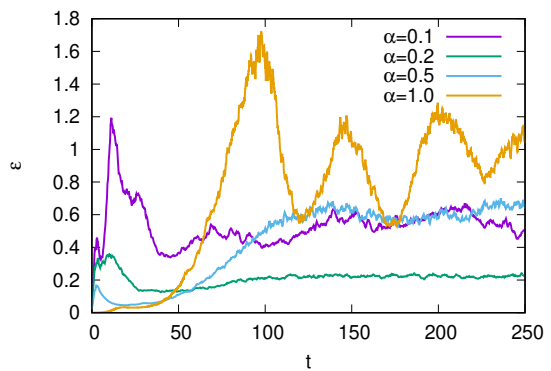

(b)

Figure 4. Time signals of (a) kinetic energy and (b) dissipation rate, averaged over the domain, for $R e=11410$ (SVV runs). The values of $\alpha$ are the same as in Figs. 2 and 3.

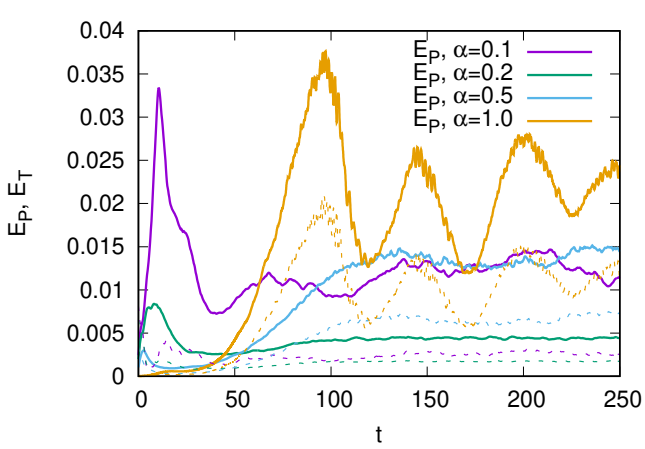

(a)

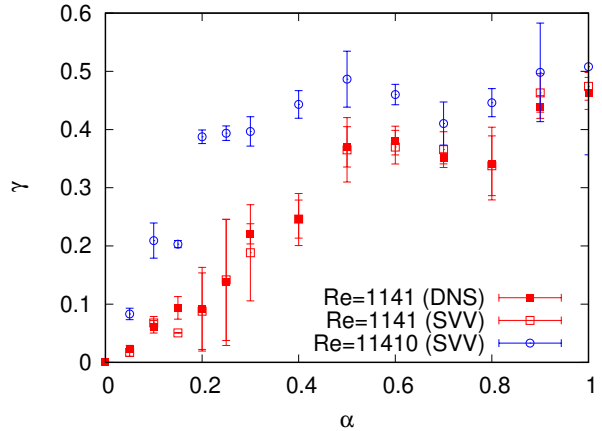

(b)

Figure 5. (a) Time signals of the toroidal and poloidal components of the kinetic energy. Poloidal energy is indicated as solid lines, toroidal energy as dotted lines. (b) Ratio $\gamma=E_{T} / E_{P}$ time-averaged in the statistically steady state, as a function of $\alpha$, for $R e=1141$ and 11410 . For the lower value of $R e$, both SVV (closed symbols) and DNS (open symbols) data are shown. For $R e=11410$, results are obtained using the SVV method only.

The origin of the quasi-periodic modulation observed for the results at $\alpha=1$ is not clear. However, we mention in this context that similar quasi-periodic fluctuations in fully developed turbulence are also observed in controlled simulations performed in cubic periodic domains in the presence of a steady forcing $[43,44]$. A further investigation of this modulation definitely seems to be an interesting perspective.

\subsection{Flow anisotropy}

After this evaluation of the global energy and dissipation balance, we now investigate how the energy is distributed over the toroidal and poloidal components. The time behavior of both components is shown for four values of $\alpha$ in Fig. 5(a). The same qualitative behavior is observed for these quantities as for the total energy and dissipation (Fig. 4). Furthermore, it is observed that the poloidal component of the energy is in all cases larger than the toroidal one. Averaging over the steady state of the simulations allows to compare quantitatively the magnitudes of both components. The ratio between the toroidal and poloidal energy components $\gamma$, defined in Eq. (7) and investigated in [26] is shown in In Fig. 5(b) as a function of $\alpha$. The figure shows that for the two Reynolds numbers considered a transition is observed from a purely poloidal flow $(\gamma=0)$, at $\alpha=0$, to an almost isotropic energy distribution $(\gamma=1 / 2$ 


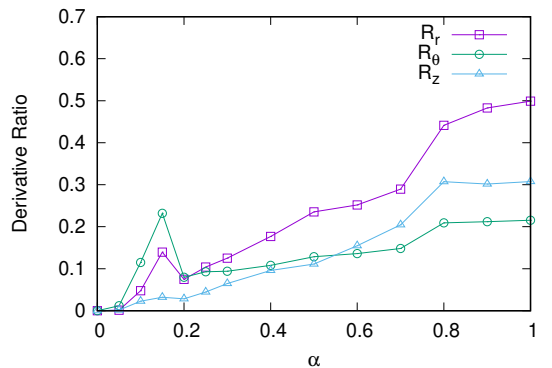

(a)

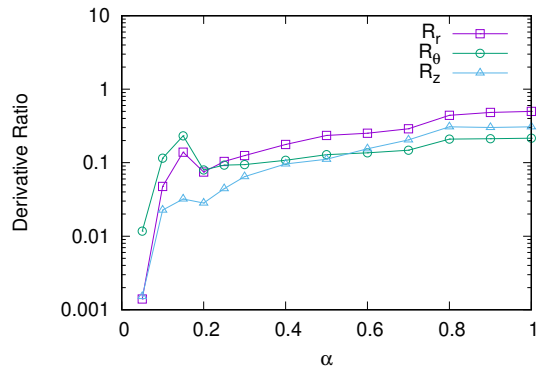

(b)

Figure 6. Anisotropy indicators of the flow in (a) linear and (b) logarithmic scale.

or, equivalently, $\left.E_{T}=E_{P} / 2\right)$ for $\alpha \rightarrow 1$. The general trend is similar for the two values of $R e$, even though the increase of $\gamma$ for small values of $\alpha$ is faster for the larger Reynolds number. The emerging picture is thus that the gradual introduction of non-axisymmetry allows a return to isotropy of the energy balance which is faster (in terms of $\alpha$ ) for higher Reynolds numbers.

We show for the low Reynolds number the results obtained using both the SVV approach and DNS. It is observed that the results are very close, which validates the SVV model, at least with respect to the global energy balance.

To complete this analysis of the global flow properties, we introduce gradient-based indicators to measure the axisymmetry of the flow. These indicators are defined as

$$
\mathcal{R}_{r}=\frac{\left\langle\left(\frac{1}{r} \frac{\partial u_{r}}{\partial \theta}\right)^{2}\right\rangle}{\left\langle\left(\frac{\partial u_{r}}{\partial r}\right)^{2}+\left(\frac{\partial u_{r}}{\partial z}\right)^{2}\right\rangle}, \quad \mathcal{R}_{z}=\frac{\left\langle\left(\frac{1}{r} \frac{\partial u_{z}}{\partial \theta}\right)^{2}\right\rangle}{\left\langle\left(\frac{\partial u_{z}}{\partial r}\right)^{2}+\left(\frac{\partial u_{z}}{\partial z}\right)^{2}\right\rangle}, \quad \mathcal{R}_{\theta}=\frac{\left\langle\left(\frac{1}{r} \frac{\partial u_{\theta}}{\partial \theta}\right)^{2}\right\rangle}{\left\langle\left(\frac{\partial u_{\theta}}{\partial r}\right)^{2}+\left(\frac{\partial u_{\theta}}{\partial z}\right)^{2}\right\rangle} .
$$

The quantities $\mathcal{R}_{r}, \mathcal{R}_{z}$ and $\mathcal{R}_{\theta}$ are therefore defined, for each velocity component, as the ratio between the strength of the volume-averaged azimuthal derivatives and that of the poloidal derivatives. Their values measure directly the relative azimuthal variations. Since their definitions involve derivatives, their value is more sensitive to small-scale variations than those of energy-based quantities. $\mathcal{R}_{r}, \mathcal{R}_{z}$ and $\mathcal{R}_{\theta}$ are plotted as a function of $\alpha$ in Fig. 6(a). One can note that all of them are increasing functions of $\alpha$. This confirms that the axisymmetry constraint is gradually relaxed as $\alpha$ increases. More specifically, the log-scale plot of Fig. $6(\mathrm{~b})$ shows that $\mathcal{R}_{i}(i \in\{r, \theta, z\})$ increases strongly for $\alpha<0.2$. Therefore, if the behaviors of $\mathcal{R}_{i}$ can be considered as indicators of small-scale dimensionality, then the transition from the axisymmetric to the threedimensional regime is most pronounced in the interval $\alpha \in[0,0.2]$.

\section{A statistical model for partially axisymmetric turbulence}

In our previous investigation [26] we developed a statistical model to reproduce and understand the dynamics of the toroidal and poloidal energy components in strictly axisymmetric turbulence. This model closes the equations $(8,9)$ governing the timeevolution of the poloidal and toroidal energy, respectively. In this section we will extend it to take into account the possible $\theta$ dependence of the dynamics introduced in the present investigation. The model as such is not meant to improve on any detailed engineering model available in the literature. It will, however, allow to interpret the 
global energy balance of the system and its anisotropy, and to understand how the parameter $\alpha$ modifies the flow.

\subsection{Modeling the pressure redistribution term}

Following the same methodology as in [26], we now derive a simple statistical model reproducing the system behavior.

When $\alpha \neq 0$, a new term $\Pi$ appears in equation $(8,9)$. This pressure term is expected to isotropize the system in the three-dimensional case. The simplest model representing such a redistribution is a linear relaxation-towards-isotropy model in the spirit of Rotta's phenomenology [45],

$$
\Pi=\alpha \frac{c_{\Pi}}{\tau}\left(E_{T}-\frac{1}{2} E_{P}\right) .
$$

This model relaxes the turbulence to its isotropic form where $E_{T}$ is one third of the total energy. The typical timescale is the nonlinear, or integral time, associated with the large-scale kinetic energy. The linearity in $\alpha$ in expression (20) is directly motivated by the shape of the pressure-strain correlation (14). Clearly, more sophisticated expressions may be thought of, but the present model represents at the zeroth order the physics of pressure distribution and relaxation towards isotropy. For an extended discussion of pressure-strain models we refer to reference-works on the subject $[46,47]$. Indeed, our goal is here not to develop a new pressure-strain model, but to understand how the pressure-strain correlation acts on the system in conjunction with other flow features such as energy production, transfer and dissipation.

Our simulations consider the case of purely poloidal forcing, $f_{\theta}=0, f_{r}, f_{z} \neq 0$, which was shown to display a strictly $2 \mathrm{D}$ behaviour with $E_{T}=0$ for the axisymmetric case $\alpha=0$ [26]. As was shown in [26], in this limit the transfer was equal to zero so that the dynamics were purely poloidal. In the presence of the pressure redistribution term, the kinetic energy can be redistributed over the other components.

To obtain an estimate of the energy redistribution, we consider the case without toroidal forcing $\left(c_{T}=0\right)$, while neglecting the transfer $T$. In the steady state, the toroidal energy balance then reduces to

$$
\epsilon_{T}=-\Pi
$$

Using the model expression for $\Pi[$ Eq. (20)] and a Taylor estimate of the dissipation [48],

$$
\epsilon_{T}=\frac{d_{T}}{\tau} E_{T}
$$

with $d_{T}$ a constant, we obtain for the energy ratio

$$
\gamma=\frac{1}{2} \frac{\alpha c_{\Pi} / d_{T}}{1+\alpha c_{\Pi} / d_{T}}
$$

which shows that for small values of $\alpha$ and $c_{T}=0$, the value of $\gamma$ is directly proportional to $\alpha$. 


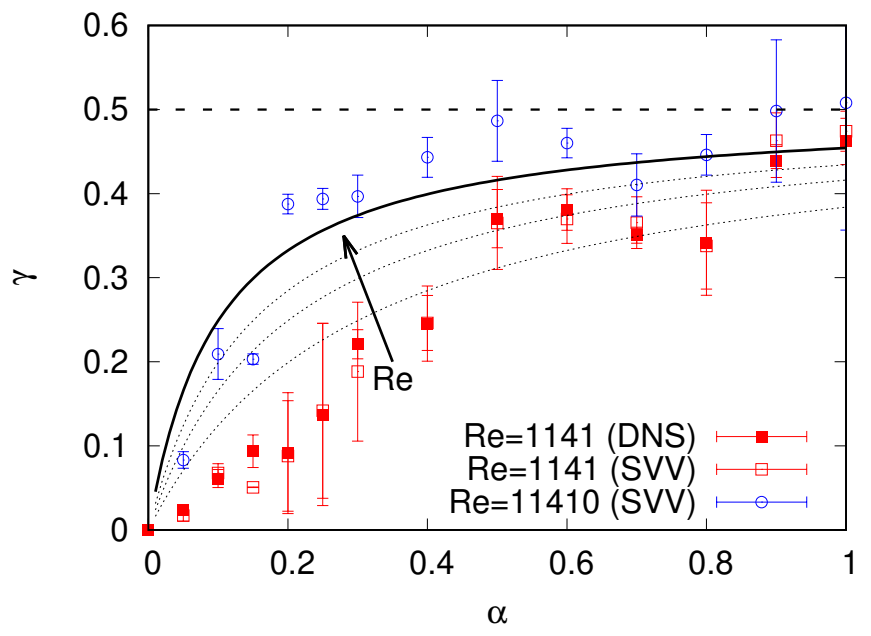

Figure 7. Comparison of the model prediction (lines) with the numerical data (symbols). The solid black line is the large Reynolds number prediction (23) with $d_{T} / c_{\Pi}=0.1$. The dotted lines are predictions obtained for smaller values of $\operatorname{Re}$ [Eq. (26), for $\left.c_{\nu} R e^{-1}=2 ; 1 ; 0.5\right]$.

The relationship (23) is plotted in Fig. 7 as a function of $\alpha$, for $d_{T} / c_{\Pi}=0.1$ (black solid line), and compared with the numerical data. This value of $d_{T} / c_{\Pi}$ was obtained by fitting the numerical data with Eq. (23). What we observe is that the model tends, as the data, to a value $\gamma=1 / 2$ for large $\alpha$. However, the model prediction shows a more rapid trend towards isotropy, in particular as compared to the low-Reynolds number simulations. Indeed, no low-Reynolds numbers effects were taken into account in the modeling approach. We now show that such effects can be taken into account in the model at first order.

At small Reynolds numbers one needs to take into account the fact that the scale separation is not sufficient and that the time-scale $\tau$ in $(22)$ is therefore affected by viscosity. In this case, we can replace Eq. (22) by

$$
\epsilon_{T} \sim d_{T}\left(\frac{1}{\tau_{\nu}}+\frac{1}{\tau_{N L}}\right) E_{T}
$$

where

$$
\tau_{\nu} \sim \frac{L^{2}}{\nu}, \quad \tau_{N L} \sim c_{P}^{-1}
$$

Using this estimate, replacing the timescale $\tau$ in the pressure strain-model (20) by $c_{P}^{-1}$ since it is an inviscid effect, and using Eq. (21), the expression for $\gamma$ becomes

$$
\gamma=\frac{1}{2} \frac{\alpha c_{\Pi} / d_{T}}{\left(1+c_{\nu} R e^{-1}\right)+\alpha c_{\Pi} / d_{T}}
$$

where $c_{\nu}$ is a model constant. Using expression (26) for arbitrary values of $R e$ allows to take into account at an empiric level the finiteness of the Reynolds number. This expression is plotted in Fig. 7 as a function of $\alpha$ for several values of $R e$ (dotted lines). The modification of the timescale allows, to a certain extent, to take into account the trends observed in the numerical data. 


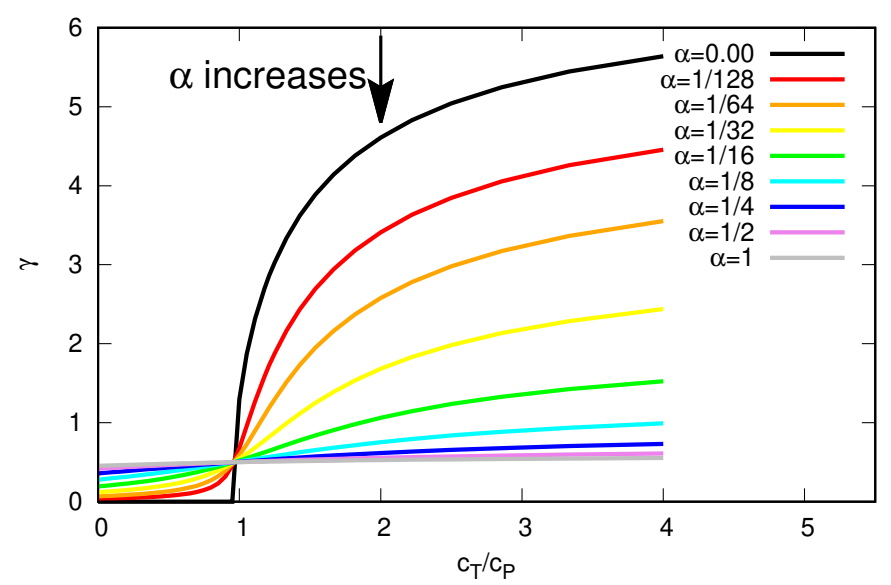

Figure 8. Model predictions of the transition from axisymmetric to non-axisymmetric flows for varying forcing anisotropy.

\subsection{Predictions of a two-equation model on the influence of forcing anisotropy}

Now that we have assessed the behavior of the pressure-strain correlation, we can adapt the two-equation model derived for purely axisymmetric turbulence [26] to the present system, in order to close the system $(8,9)$. This will allow us to assess how the transition from a purely poloidal flow towards a mixed poloidal-toroidal (or swirling) flow is affected when the forcing anisotropy is changed. In the foregoing, we have kept $c_{T}=0$ and $c_{P}=0.4$. Carrying out a full parameter scan varying simultaneously $c_{T} / c_{P}$ and $\alpha$ using DNS would be prohibitively expensive. We therefore integrate the simple energy model proposed in [26], completed with the pressure strain mode (20). We consider the high-Reynolds number case and do therefore not use the above mentioned viscous correction to the dissipation. The model is

$$
\begin{aligned}
T & =\frac{\zeta \tau E_{T}\left(E_{T}-2 E_{P}\right)}{L^{2}}, \\
P_{i} & =2 c_{i} E_{i}, \\
\varepsilon_{i} & =d_{i} \frac{E_{i}}{\tau}, \\
\Pi & =\alpha \frac{c_{\Pi}}{\tau}\left(E_{T}-\frac{1}{2} E_{P}\right),
\end{aligned}
$$

with $i \in\{T, P\}$ and $\tau$ a characteristic timescale which we have modeled as

$$
\tau=L /\left(E_{P}+\alpha E_{T}\right)^{1 / 2} .
$$

Constants are chosen $c_{\Pi}=9, d_{P}=0.9, d_{T}=0.9, \zeta=0.024$. Indeed, we have set $c_{\Pi} / d_{T}=10$ as evaluated in Fig. 7 and we have chosen the model-constants $d_{P}, d_{T}, \zeta$ as in our previous investigation, even though the forcing was different [26]. The present results are therefore meant to be qualitative.

We have numerically integrated the above model and the results are shown in Fig.8. It can be seen that when strictly axisymmetric i.e. $\alpha=0$, the flow remains stably 
poloidal $(\gamma=0)$ up to a value of $c_{T} / c_{P} \approx 1$. It was shown analytically in Ref. [26] that the model, in the absence of the pressure term displayed some hysteresis, but only in a very narrow band of values $c_{T} / c_{P}$. We do not focus on this effect here which is absent as soon as $\alpha$ is not negligably small anymore. Instead, we focus on the robustness of the poloidal regime as a function of $\alpha$.

When $\alpha$ increases from 0 to $1 / 128$, the values of $\gamma$ depart visibly from the case of $\alpha=0$, but there is still a significant range of $c_{T} / c_{P}$ where the flow remains close to purely poloidal. The larger values of $\alpha$ show that the transition between the $\alpha=0$ and the $\alpha=1$ case is gradual. The results remain obviously of speculative nature, but it seems plausible that qualitatively the pressure-strain redistribution will act as illustrated by the model.

\section{Conclusion}

The here presented numerical simulations illustrate how turbulence in a cylindrical cavity behaves, when the flow is partially axisymmetric. The results confirm that the flow remains purely poloidal when axisymmetry is imposed, as was observed in ref. [26]. However, as soon as the axisymmetry is broken, pressure-strain correlations allow to redistribute energy to the toroidal component. For small three-dimensionality (small $\alpha$ ), the flow remains close to poloidal, and the ratio of toroidal to poloidal energy $\gamma$ is a linear function of $\alpha$ in this limit. It therefore seems that an almost purely poloidal flow can survive in close to axisymmetric flow. A possible application of this case is the tokamak, a thermo-nuclear fusion plasma geometry, where the toroidal magnetic field is very strong leading to close to axisymmetric dynamics. Possibly in such a flow a transition from purely poloidal to three-component flow might be observed. We therefore currently investigate a flow similar to the current one in toroidal geometry.

In this context it will be interesting to investigate the other limit where $\alpha \rightarrow \infty$. Such a case is interesting since physically it might correspond to the case where diffusion and viscosity are dominant along the toroidal direction, leading thereby to a flow which is axisymmetric through the rapid smoothening of variations in that direction. In this limit the resulting flow might thereby also be close to axisymmetric, but the underlying mechanisms leading to the state would be radically different. This is left for future research.

\section{Acknowledgments}

Eric Serre is gratefully acknowledged for providing the numerical code. ZQ was supported by a grant from the China Scholarship Council. All simulations were carried out using the facilities of the PMCS2I (École Centrale de Lyon). Laurent Pouilloux and Dan-Gabriel Calugaru are acknowledged for technical assistance.

\section{Disclosure statement}

No potential conflict of interest was reported by the author(s). 


\section{References}

[1] H.P. Greenspan The theory of rotating fluids. CUP Archive, 1968.

[2] H.K. Moffatt. On the suppression of turbulence by a uniform magnetic field. J. Fluid Mech., 28, 1967.

[3] B. Gallet. Exact two-dimensionalization of rapidly rotating large-Reynolds-number flows. J. Fluid Mech., 783:412-447, 2015.

[4] B. Gallet and C.R. Doering. Exact two-dimensionalization of low-magnetic-Reynoldsnumber flows subject to a strong magnetic field. J. Fluid Mech., 773:154-177, 2015.

[5] A. Delache, C. Cambon, and F. Godeferd. Scale by scale anisotropy in freely decaying rotating turbulence. Phys. Fluids, 26:025104, 2014.

[6] B. Favier, F. S. Godeferd, C. Cambon, and A. Delache. On the two-dimensionalization of quasistatic magnetohydrodynamic turbulence. Phys. Fluids, 22:075104, 2010.

[7] B. Bigot and S. Galtier. Two-dimensional state in driven magnetohydrodynamic turbulence. Phys. Rev. E, 83:026405, 2011.

[8] A. Celani, S. Musacchio, and D. Vincenzi. Turbulence in more than two and less than three dimensions. Phys. Rev. Lett., 104:184506, 2010.

[9] H. Xia, D. Byrne, G. Falkovich, and M. Shats. Upscale energy transfer in thick turbulent fluid layers. Nature Phys., 7:321, 2011.

[10] S.J. Benavides and A. Alexakis. Critical transitions in thin layer turbulence. J. Fluid Mech., 822:364, 2017.

[11] B. Favier, L.J. Silvers, and M.R.E. Proctor. Inverse cascade and symmetry breaking in rapidly rotating boussinesq convection. Phys. Fluids, 26:096605, 2014.

[12] N. Leprovost, B. Dubrulle, and P.-H. Chavanis. Dynamics and thermodynamics of axisymmetric flows: Theory. Phys. Rev. E, 73:046308, 2006.

[13] L. Onsager. Statistical hydrodynamics. Il Nuovo Cimento, 6:279, 1949.

[14] D. Montgomery, W.H. Matthaeus, W. T. Stribling, D. Martinez, and S. Oughton. Relaxation in two dimensions and the "sinh-Poisson" equation. Phys. Fluids A, 4:3, 1992.

[15] R. Robert and J. Sommeria. Statistical equilibrium states for two-dimensional flows. $J$. Fluid Mech., 229:291, 1991.

[16] J. Miller. Statistical mechanics of Euler equations in two dimensions. Phys. Rev. Lett., 65:2137, 1990.

[17] K. Mohseni. Statistical equilibrium theory for axisymmetric flows: Kelvin's variational principle and an explanation for the vortex ring pinch-off process. Phys. Fluids, 13:1924, 2001.

[18] R. Monchaux, F. Ravelet, B. Dubrulle, A. Chiffaudel, and F. Daviaud. Properties of steady states in turbulent axisymmetric flows. Phys. Rev. Lett., 96:124502, 2006.

[19] R. Monchaux, P.-P. Cortet, P.-H. Chavanis, A. Chiffaudel, F. Daviaud, P. Diribarne, and B. Dubrulle. Fluctuation-dissipation relations and statistical temperatures in a turbulent von Kármán flow. Phys. Rev. Lett., 101(17):174502, 2008.

[20] A. Naso, S. Thalabard, G. Collette, P.-H. Chavanis, and B. Dubrulle. Statistical mechanics of Beltrami flows in axisymmetric geometry: Equilibria and bifurcations. J. Stat. Mech., 2010:P06019, 2010.

[21] A. Naso, R. Monchaux, P.H. Chavanis, and B. Dubrulle. Statistical mechanics of Beltrami flows in axisymmetric geometry: Theory reexamined. Phys. Rev. E, 81:066318, 2010.

[22] S. Thalabard, B. Dubrulle, and F. Bouchet. Statistical mechanics of the 3d axisymmetric Euler equations in a Taylor-Couette geometry. J. Stat. Mech., 2014:P01005, 2014.

[23] S. Thalabard, B. Saint-Michel, E. Herbert, F. Daviaud, and B. Dubrulle. A statistical mechanics framework for the large-scale structure of turbulent von Karman flows. New J. Phys., 17:063006, 2015.

[24] B. Qu, W.J.T. Bos, and A. Naso. Direct numerical simulation of axisymmetric turbulence. Phys. Rev. Fluids, 2:094608, 2017.

[25] B. Qu, A. Naso, and W.J.T. Bos. Cascades of energy and helicity in axisymmetric turbulence. Phys. Rev. Fluids, 3:014607, 2018. 
[26] Z. Qin, H. Faller, B. Dubrulle, A. Naso, and W.J.T. Bos. Transition from non-swirling to swirling axisymmetric turbulence. Phys. Rev. Fluids, 5(6):064602, 2020.

[27] U. Frisch, M. Lesieur, and P.L. Sulem. Crossover dimensions for fully developed turbulence. Phys. Rev. Lett., 37:1312, 1976.

[28] J.-D. Fournier and U. Frisch. d-dimensional turbulence. Phys. Rev. A, 17:747, 1978.

[29] U. Frisch, A. Pomyalov, I. Procaccia, and S.S. Ray. Turbulence in noninteger dimensions by fractal fourier decimation. Phys. Rev. Lett., 108:074501, 2012.

[30] A.S. Lanotte, R. Benzi, S.K. Malapaka, F. Toschi, and L. Biferale. Turbulence on a fractal fourier set. Phys. Rev. Lett., 115:264502, 2015.

[31] W.J.T. Bos, F. Laadhari, and W. Agoua. Linearly forced turbulence at low Reynolds numbers. Phys. Rev. E, 102:033105, 2020.

[32] I. Raspo, S. Hugues, E. Serre, A. Randriamampianina, and P. Bontoux. A spectral projection method for the simulation of complex three-dimensional rotating flows. Computers Ef fluids, 31:745, 2002.

[33] N. Peres, S. Poncet, and E. Serre. A 3d pseudospectral method for cylindrical coordinates. Application to the simulations of rotating cavity flows. J. Comp. Phys., 231:6290, 2012.

[34] W. Heinrichs. Spectral collocation schemes on the unit disc. J. Comp. Phys.., 199:66, 2004.

[35] Z. Qin. Transitions in Axisymmetric Turbulence. PhD thesis, Université de Lyon, 2019.

[36] T. Kato. Remarks on zero viscosity limit for nonstationary navier-stokes flows with boundary. In Seminar on nonlinear partial differential equations, pages 85-98. Springer, 1984.

[37] R. Nguyen van Yen, M. Farge, and K. Schneider. Energy dissipating structures produced by walls in two-dimensional flows at vanishing viscosity. Phys. Rev. Lett., 106:184502, 2011.

[38] E. Tadmor. Convergence of spectral methods for nonlinear conservation laws. SIAM J. Num. Analysis, 26:30-44, 1989.

[39] E. Séverac and E. Serre. A spectral vanishing viscosity for the les of turbulent flows within rotating cavities. J. Comput. Phys., 226:1234-1255, 2007.

[40] T.S. Lundgren. Linearly forced isotropic turbulence. Annual Research Briefs-2003, p.461, 2003.

[41] C. Rosales and C. Meneveau. Linear forcing in numerical simulations of isotropic turbulence: Physical space implementations and convergence properties. Phys. Fluids, 17:095106, 2005.

[42] F. Ravelet, A. Chiffaudel, F. Daviaud, and J. Léorat. Toward an experimental von Kármán dynamo: Numerical studies for an optimized design. Phys. Fluids, 17:117104, 2005.

[43] T. Yasuda, S. Goto, and G. Kawahara. Quasi-cyclic evolution of turbulence driven by a steady force in a periodic cube. Fluid Dyn. Res., 46:061413, 2014.

[44] S. Goto, Y. Saito, and G. Kawahara. Hierarchy of antiparallel vortex tubes in spatially periodic turbulence at high Reynolds numbers. Phys. Rev. Fluids, 2:064603, 2017.

[45] J.C. Rotta. Statistische Theorie nichthomogener Turbulenz. Z. Phys., 129:547, 1951.

[46] S.B. Pope. Turbulent Flows. Cambridge University Press, 2000.

[47] P. Sagaut and C. Cambon. Homogeneous Turbulence Dynamics. Cambridge University Press, 2008.

[48] G. I. Taylor. Statistical theory of turbulence. Proc. Roy. Soc. London. Ser. A, Math. Phys. Sci., 151:421, 1935. 